International Journal of Engineering \& Technology, $7(2.13)(2018) 247-251$
SPC
International Journal of Engineering \& Technology
Website: www.sciencepubco.com/index.php/IJET
Research Paper

\title{
theoretical bases of perspective chemical sources of current (CSC) and ultra-high-volume capacitor structures (UCS) for electric energy accumulators
}

\author{
Sleptsov Vladimir Vladimirovich ${ }^{1 *}$, Kukushkin Dmitry Yuryevich ${ }^{2}$, Diteleva Anna Olegovna ${ }^{2}$ \\ ${ }^{1}$ Doctor of Engineering Sciences (Advanced Doctor), Professor, Moscow Aviation Institute \\ ${ }^{2}$ Assistant Professor, Moscow Aviation Institute \\ *Corresponding author E-mail:
}

\begin{abstract}
The paper analyzes thin-film technologies for advanced CSC and UCS. It is shown that the amount of accumulated energy is determined by the work of moving the charges in a given direction. This dependence determines the energy intensity of the CSC and UCS shows that the process of accumulation of electric energy in them is the same, and is determined mainly by polarization effects in the system. Because the mechanism of accumulation of electric energy in the CSC and UCS from a physical point of view is the same, there is a possibility to combine design solutions and to create a unified electrode material for a CSC and UCS in thin-film technology. In this case, it is possible to increase the specific energy capacity of the energy storage device while increasing its specific power in a single design solution. The traditional design of CSC and electrolytic UCS focused on the formation of electrode materials for thick-film technology. From this point of view, thin-film technologies, which have been actively developed recently, are promising. The prospect of developing thin-film technologies is associated with the use of nanomaterials and nanostructures, which can form more energy-intensive materials and significantly change the processes of converting the energy of the double electric layer and chemical interaction into electrical energy. The task of the article is to study thin-film technologies theoretically and experimentally in order to determine their development prospects for high-energy current sources, determine the ranges of their use and the possibility of industrial implementation. However, recently there has been a tendency to reduce the dynamics of growth in energy intensity of CSC generated by this technology.
\end{abstract}

Keywords: Thin Film Technology; Chemical Power Source; Ultra-High-Volume Capacitor Structures; Electric Energy Accumulators; Energy Consumption; Electrode Materials; Battery; Mechanism of Electric Energy Accumulation; Electrolyte.

\section{Introduction}

In the work, the analysis of thin-film technologies for perspective CSC and UCS is carried out. It is shown that the value of the accumulated energy is determined by the work of moving the charges in a given direction and is expressed by the formula: $\mathrm{W}=\mathrm{PE}$, where $\mathrm{W}$ the work, $\mathrm{P}$ - the dipole moment, $\mathrm{E}$ - the electric field. This dependence determines the energy intensity of the UCS and CSC, shows that the process of electric energy accumulation in them is the same, and is determined mainly by polarization effects in the system. Due to the fact that the mechanism of electric energy accumulation in the UCS and CSC from a physical point of view is the same, there is a possibility to combine design solutions and to create a unified electrode material for UCS and CSC on thin-film technology. In this case, it is possible to increase the specific energy capacity of the energy storage device while increasing its specific power in a single design solution. Therefore, the development of advanced design of UCS and SCS should go in the following directions:

The unified nanostructured electrode material development for USC and CSC;

The nanostructured active mass development for CSC, ceramics and polymer and ceramic materials with dielectric permeability

$\varepsilon>10^{6} \div 10^{8}$ And $\mathrm{U}_{\mathrm{br}}>0,01 \div 0,1 \frac{\mathrm{MV}}{\mathrm{m}}$
The development of new electrolytes, providing high $\boldsymbol{E}_{S}$ in DEL. Current trends in the development of the global renewable energy market, electronics and instrument making medicine and a number of other areas of science and technology indicate a steady increase in the share of autonomous power sources. The relevant problem of autonomous power supply of high-current consumers has not been completely solved. The creation of such devices and industrial technologies and their production will allow obtaining breakthrough results in such areas as electric transport, power recovery systems, generators based on renewable energy sources, uninterruptible power supplies, etc.

Today the best examples of battery - a secondary chemical sources of current (CSC), which provide the energy consumption of about $250(\mathrm{~W} \cdot \mathrm{h}) / \mathrm{kg}$ and the intensive research on creation in the next 5 years energy storage with energy up to $450-500(\mathrm{~W} \cdot \mathrm{h}) / \mathrm{kg}$, and in the distant prospect, and to theory-based limit $1000-1200$ $(\mathrm{W} \cdot \mathrm{h}) / \mathrm{kg}$ is carried out. The energy consumption of ultra-high-volume capacitor structures (UCS) 10-20 (W/h)/ $\mathrm{kg}$, the intensive research to achieve specific energy intensity $20-40(\mathrm{~W} \cdot \mathrm{h}) / \mathrm{kg}$, and for hybrid UCS $220-250(\mathrm{~W} \cdot \mathrm{h}) / \mathrm{kg}$ is carried out.

The traditional design of the CSC and electrolytic UCS is focused on the formation of electrode materials on thick-film technology, when the chemically active substance for the CSC and superporous material for UCS are applied in a thick layer $(30-60 \mathrm{mkm})$ on aluminum or copper foil in the form of paste. This is quite cheap and 
productive technology is used widely for industrial purposes. However, recently there has been a downward trend in the growth of the energy intensity dynamics of the CSC, created by this technology [1-3]. Therefore, the urgent task is to find and develop new technologies that will provide the necessary dynamics of energy intensity growth of CSC and UCS. From this point of view, thin-film technologies, which have been actively developed recently, become promising [4-6]. The prospect of developing thin-film technologies is associated with the use of nanomaterials and nanostructures, which can form more energy-intensive materials and change significantly the processes of converting the energy of the double electric layer (DEL) and chemical interaction into electrical energy. However, the prospects for the development of thin-film technologies are considered for low-energy CSC, which are used for smart cards, chips with built-in power supplies for portable devices of various applications, medical implants, etc. [4-5]

The task of the article is to study theoretically and experimentally thin-film technologies in order to determine their prospects development for high-energy current sources, determine the ranges of their use and the possibility of industrial implementation.

\section{Theoretical study of thin-film technology of formation of electrode materials for CSC and electrolytic UCS}

In accordance with the generalized equation of the first and second laws of thermodynamics of energy, supply and drainage in the system can be carried out only in two ways. It is possible to supply and take away energy in the form of heat or in the form of work. Mathematically, this statement is written as follows [7-9]:

$\mathrm{E}=\mathrm{T} \cdot \mathrm{S} \pm \sum_{\mathrm{i}=0}^{\mathrm{n}} \mathrm{A}_{\mathrm{i}}$

where $\mathrm{E}$ - energy supplied to the system, $\mathrm{T}$ - temperature of the system, $\mathrm{S}$ - entropy of the system, $-A_{i}$ - work produced by the system (when energy is taken away) or $+A_{i}$, when energy is supplied to the system.

For real irreversible processes work that can be supplied to the system or extracted from the system is written as a multiplication of two quantities - extensive (X) and intensive (Y):

$A_{i}=X \cdot y$

In this case, equation (1) should be written in the following form:

$d E=d(T \cdot S)+d(P \cdot V)+(\delta \cdot F)+d(\varphi \cdot q)+d \sum\left(\mu_{i} n_{i}\right)$

Where $\mathrm{T}$ - temperature, $\mathrm{P}$ - pressure, $\delta$ - surface energy, $\varphi$ - electric potential, $\mu_{\mathrm{i}}$-chemical potential -

extensive parameters, $\mathrm{S}$ - entropy, $\mathrm{V}$ - volume, $\mathrm{q}$ -

charge, $n_{i}$ - the number of moles - intensive parameters.

If extensive parameters with decreasing structural elements to nanoscale values start to depend on intensive ones, equation (3) can be written as [10]:

$\mathrm{dE}=\left(\frac{\partial \mathrm{S}}{\partial \mathrm{T}} \cdot \frac{\mathrm{dT}}{\mathrm{dr}} \cdot \frac{\partial \mathrm{T}}{\partial \mathrm{S}} \cdot \frac{\mathrm{dS}}{\mathrm{dr}}\right)$

$\mathrm{dE}=\left(\frac{\partial \mathrm{S}}{\partial \mathrm{T}} \cdot \frac{\mathrm{dT}}{\mathrm{dr}}+\frac{\partial \mathrm{T}}{\partial \mathrm{S}} \cdot \frac{\mathrm{dS}}{\mathrm{dr}}\right) \mathrm{dr}+\left(\frac{\partial \mathrm{P}}{\partial \mathrm{V}} \cdot \frac{\mathrm{dV}}{\mathrm{dr}}+\frac{\partial \mathrm{V}}{\partial \mathrm{P}} \cdot \frac{\mathrm{dP}}{\mathrm{dr}}\right) \mathrm{dr}+\left(\frac{\partial \delta}{\partial \mathrm{F}} \cdot \frac{\mathrm{dF}}{\mathrm{dr}}+\right.$ $\left.\frac{\partial \mathrm{F}}{\partial \delta} \cdot \frac{\mathrm{d} \delta}{\mathrm{dr}}\right) \mathrm{dr}+$

$+\left(\frac{\partial \varphi}{\partial \mathrm{q}} \cdot \frac{\mathrm{dq}}{\mathrm{dr}}+\frac{\partial \mathrm{q}}{\partial \varphi} \cdot \frac{\mathrm{d} \varphi}{\mathrm{dr}}\right) \mathrm{dr}+\sum_{\mathrm{i}=1}^{\mathrm{n}}\left(\frac{\partial \mu_{\mathrm{i}}}{\partial \mathrm{n}_{\mathrm{i}}} \cdot \frac{\mathrm{dn}_{\mathrm{i}}}{\mathrm{dr}}+\frac{\partial \mathrm{n}_{\mathrm{i}}}{\partial \mu_{\mathrm{i}}} \cdot \frac{\mathrm{d} \mu_{\mathrm{i}}}{\mathrm{dr}}\right) \mathrm{dr}$

In this case, the dependence of extensive and intensive parameters on the dimensional factor appears in full differential, which changes significantly the energy intensity of the system as a whole.
Equation (4) leads to the conclusion that nanostructured systems are fundamentally different in energy intensity from monolithic materials, and numerous experiments confirm clearly this theoretical result. [11-12]

The analysis (4) shows that as a result of Nano structuring, which is provided by thin-film technology, the energy storage mechanisms arise in the system not only due to chemical reactions, but also due to the formation of charges at the boundaries, as well as other possible types of work that can go into electrical work if there is an appropriate mechanism. As a rule, there are always more energy inhomogeneities at the boundaries of areas, which ensure the functioning of such mechanisms. Therefore, nanostructuring of the material (the formation of a huge number of boundaries of the areas) on the one hand creates a significantly greater energy reserve in comparison with monolithic materials, and on the other hand, allows creating mechanisms that convert different types of energy into electrical

In the work [13] it is shown that thermodynamic and electrostatic account of polarization interactions of particles in system leads to the same result - chemical potential of a molecule surrounded by the neighbors creating some effective field $\mathrm{E}$ is equal to work of polarization of the considered molecule. Thus, the universal nature of electrochemical reactions that convert chemical energy into electrical energy is based on polarization effects.

In this case, the chemical potential of the molecule (up to additive constant KT, independent of the electric field intensity) can be represented as:

$\mu=-P E=A_{i}$

Where $\mathrm{P}$ - dipole moment, $\mathrm{E}$ - electric field strength, $\mathrm{A}_{\mathrm{i}}$ - electric field work.

Formula (5) as a result of simple transformations can be written in the form:

$\delta A_{i}=-\operatorname{ed} \varphi$

In this case, Gibbs potential in isobaric and isothermal conditions can be recorded as [13]:

$\mathrm{dG}_{\mathrm{PV}}=\sum \mathrm{N}_{\mathrm{i}} \mathrm{d} \mu_{\mathrm{i}}=\sum \mathrm{N}_{\mathrm{i}} \mathrm{ed} \varphi=\sum \mathrm{N}_{\mathrm{i}} \mathrm{Edp}_{\mathrm{i}}$

Each of the expressions (7) is a multiplication of an intensive quantity on extensive one. As extensive quqantity is the density of molecules $\left(\boldsymbol{N}_{i}\right)$ and as intensive one - the chemical potential, electric work free $(\mathrm{ed} \varphi)$ or related charges (pdE).

Taking into account the electrocapillary phenomena that occur in the formation of the surface of the section of two adjacent phases, Gibbs potential is recorded as follows:

$\mathrm{dG}_{\mathrm{S}}=\delta \mathrm{dF}+\mathrm{Edp}$

Where $\mathrm{E}$ - electric field strength, $\mathrm{P}$ - dipole moment, $\delta$ - surface tension, F - surface area.

Using Maxwell's transformation and Poisson's equation for equation (8), we obtain the fundamental equation of electrocapillarity [13-16]:

$\frac{\partial \delta}{\partial x}=-\rho^{\text {free }}+\rho^{\text {bound }}=-q$

Where $\rho^{\text {freе }}$ и $\rho^{\text {bound }}$ - surface density of free and bound charges,

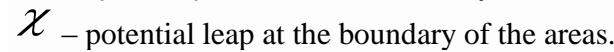

Under free charge all electric charges are understood, which under the influence of the electric field can be moved to macroscopic distances. Equation (9) is called the first Lippman equation. Charges that are part of molecules, dipoles, etc. are called bound. At the point of electrocapillary maximum

$\delta^{\text {free }}+\delta^{\text {bound }}$ 
This makes it possible to quantify the chemical potential of the area in contact with the metal [13], [16]. Formula (9) is obtained strictly thermodynamically and contains no assumptions or model representations. The first part is a full charge of the surface layer, located in the averaged electrostatic field E. The left side of equation (9) shows that the stable state of the boundary is achieved by compensating the forces of electrostatic and non-electrostatic origin. No restrictions were applied at the derivation of equation (9). So it is true in the description of any phase boundaries, which arise when CSC and UCS working. Equation (10) makes it possible to quantify the chemical potential of the active area in contact with the metal [1316]. Due to the fact that the chemical potential $\mu$ is equal to PE, the multiplication of the dipole moment on the effective field:

$\mu=-\mathrm{PE}$

Equation (11) leads to the conclusion that the chemical potential of a molecule surrounded by its neighbors, creating some effective field $\mathrm{E}$, is equal to the polarization of the molecule.

$A_{n}=p E=\mu$

Where $A_{n}$-the polarization work of a molecule.

Differential of equation (13) can be written as

$\mathrm{dA}_{\mathrm{n}}=\mathrm{pdE}+\mathrm{Edp}=\mathrm{d} \mu$

According to the equation of the first and second laws of thermodynamics can be written as $|7|$

$\mathrm{dG}_{(\mathrm{dT}=0, \mathrm{dp}=0)}=\sum \mathrm{N}_{\mathrm{i}} \mathrm{d} \mu_{\mathrm{i}}$

\section{Correlation}

$\mathrm{dG}=\sum\left[\mathrm{N}_{-} \mathrm{i} \mathrm{d} \mu \_\mathrm{i}=\mathrm{N} \_\mathrm{i}\right.$ ed $\left.\Delta \varphi\right)$

Is widely used in the theory of galvanic elements that convert, first of all, the energy of a chemical reaction into electrical energy [13 16].

In the integral form this equation can be written as follows

$$
\Delta G_{\text {chem.r. }}^{\circ}=\sum \Delta G_{\text {fcon }}^{\circ}-G_{\text {fout }}^{\circ}=\sum N_{i} \mu_{\text {con }}^{\circ}-\sum N_{i} \mu_{\text {out }}^{\circ}=\mathrm{z} \varepsilon
$$

Where $\mathrm{z}=\sum \mathrm{N}_{\mathrm{i}} \mathrm{e}-$ the electricity amount (charge) passed through the external circuit of the galvanic element. This charge as follows from equation (16) is proportional to the amount of the substance reacted in the element,

$\mathrm{EMF}=\sum \Delta \varphi_{\mathrm{i}}-$ it is the electromotive force (EMF) of the element equal to the sum of leaps of potentials $\sum \Delta \varphi_{\mathrm{i}} \Phi$. In this case, the EMF of the element in the first approximation can be estimated as

$$
\mathrm{EMF}=\frac{\Delta \mathrm{G}_{\text {chem.r. }}^{\circ}}{\mathrm{z}}
$$

Equation (15) considers the situation when only the processes associated with the movement of constant dipole moments occur in the system. This is explained by the fact that at room temperatures and voltages up to 10 volts, the contribution to the polarization from induced dipole moments is about 100 times less than from constant ones [17].

Therefore, in a complete differential at the boundaries of smooth surfaces, the value of EdP in equation (8) is usually neglected. However, at the nanostructured interface of media, where the electric field intensity can exceed $10^{6} \div 10^{7} \mathrm{~V} / \mathrm{sm}$. [13], [16], the contribution to the general polarization of induced dipole moments will be more significant. It should also be noted that the equations (16, 17) allow concluding that thin-film structures have less thermal losses associated with the passage of current through the external circuit of the galvanic element due to the flow of unrelated charges due to the fact that the thickness of the chemically active layer is more than two orders of magnitude less.

\section{Systems without chemical reactions (ultra- high volume capacitors electrolytic struc- tures).}

The key parameter of systems that accumulate electric energy without chemical reactions is the electric capacity. The value of the differential capacity can be found using electrocapillary curves. [13$16]$

$\frac{\mathrm{d}^{2} \delta}{\mathrm{d} \varphi^{2}}=\frac{\mathrm{dq}}{\mathrm{d} \varphi}=\mathrm{C}_{\mathrm{d}}$

The expression (18) is called the second Lippman equation.

Equation (18) can be represented as follows:

$\mathrm{dq}=\mathrm{C}_{\mathrm{d}} \mathrm{d} \varphi$

Integration of the equation (19) gives an integral capacity C. For a flat capacitor:

$$
C=\frac{\varepsilon_{0} \varepsilon S}{d}
$$

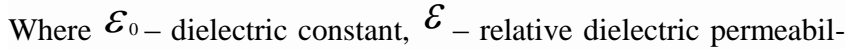
ity, $\mathrm{S}$ - the area of the capacitor plates, $\mathrm{d}$ - the distance between the plates [13-17]. The energy stored in the capacitor structure according to the square dependence increases with increasing voltage in the capacitor:

$\mathrm{E}=\frac{\mathrm{CU}^{2}}{2}$

In the work [13], [16], [17] the influence of bound and free charges on the capacity of DEL was shown, which were called dipole and electronic components, respectively. The Lippmann equation can be converted to form:

$$
\frac{\mathrm{d} \varphi}{\mathrm{d} \rho}=\frac{\mathrm{d} \varphi_{\mathrm{e}}}{\mathrm{d} \rho}+\frac{\mathrm{d} \varphi_{\mathrm{d}}}{\mathrm{d} \rho} \text { или } \mathrm{C}^{-1}=\mathrm{C}_{\mathrm{e}}^{-1}+\mathrm{C}_{\mathrm{d}}^{-1}
$$

Where

$\mathrm{C}_{\mathrm{e}}^{-1}-$ the electronic part of DEL capacity,

$\mathrm{C}_{\mathrm{d}}^{-1}$ - dipole part of DEL capacity.

In this case, the expression for the electronic part of the DEL capacity can be represented as:

$\mathrm{C}_{\mathrm{e}}=\frac{\mathrm{d} \rho}{\mathrm{d} \Delta \mathrm{x}}=\frac{\mathrm{dp} \mathrm{e}}{\mathrm{dz}} \cdot \frac{\mathrm{dz}}{\mathrm{d} \Delta \mathrm{x}}=\frac{\mathrm{p}_{\mathrm{e}}^{\mathrm{V}}}{\mathrm{p}_{\mathrm{e}}^{\mathrm{S}}} \cdot \frac{\varepsilon_{\mathrm{S}}}{4 \pi}$

Where $\mathrm{p}_{\mathrm{e}}^{\mathrm{V}}$ and $\mathrm{p}_{\mathrm{e}}^{\mathrm{S}}$ respectively the bulk and the surface density of free charge, $\mathrm{z}$ - DEL thickness, $\Delta \chi-$ the potential leap at the boundary, $\varepsilon_{S}-$ DEL dielectric permeability.

The equation (23) allows drawing a conclusion at once that it is necessary to use substances with high electrical conductivity as electrode material.

The second term in equation (22) is written as follows:

$\frac{d \rho}{d \varphi_{d}}=\frac{d \rho}{d \rho_{d}} \cdot \frac{d \rho d}{d \varphi d}=\bar{\varepsilon} \frac{d \rho d}{d \varphi}=C_{d}$

Where $\frac{\mathrm{d} \rho}{\mathrm{d} \rho \mathrm{d}}=\bar{\varepsilon}-$ const, i.e., it represents the effective dielectric permeability averaged over the whole dipole layer.

The equation (24) leads to the conclusion that the DEL capacity increases with the increase in the number of dipoles and dipole moment of DEL, which ensures the growth $\varepsilon_{\mathrm{s}}$ and the factor $\frac{\mathrm{d} \rho \mathrm{d}}{\mathrm{d} \varphi}$. The equations (20) and (23) include one very important parameter that 
determines the operating voltage of the CSC and a number of UCS, which is called the potential leap at the boundary ( $\Delta \chi)$. Phenomenological consideration of the electrostatics boundary $[13,16$,$] al-$ lowed obtaining an analytical expression to determine the leap of the potential:

$$
\Delta \mathrm{x}=\frac{\mathrm{W}}{\mathrm{e}}-\frac{\mathrm{K}_{\mathrm{B}} \mathrm{T}}{\mathrm{e}} \ln \frac{2 \pi \mathrm{e}^{2}}{\left(3 \pi^{2}\right)^{\frac{1}{3} \varepsilon}} \cdot \sqrt{\frac{2 \pi \mathrm{m}}{\mathrm{n}^{2} \mathrm{~K}_{\mathrm{B}}}}+\text { const }
$$

Where $\mathrm{W}$ - the electron output work, $\boldsymbol{E}_{-}$DEL dielectric permeability, $\mathrm{m}$-electron mass.

\section{Consideration of results, conclusions and statement of the problem.}

1) As a result of the analysis of accumulation mechanisms of electric energy in CSC and UCS it is shown that in both cases process of accumulation of electric energy is connected with change of energy of free and connected charges. The amount of accumulated energy is determined by the work of moving the charges in a given direction and is expressed by the formula: $\mathrm{W}=\mathrm{PE}$, where $\mathrm{W}$ - work, $\mathrm{P}$ - dipole moment, $\mathrm{E}$ - electric field. This dependence determines the energy capacity of CSC and UCS. The analysis showed that the process of accumulation of electric energy in CSC and UCS is the same and is determined mainly by polarization effects in the system.

2) From the equation [25], it follows that the surface potential leap, which determines the operating voltage of the CSC and electrolytic UCS (ignitors) with an accuracy of a constant is determined by the electron output work to the environment. This formula, obtained on the basis of quantum-mechanical representations, is consistent with thermodynamic approaches to the definition of $\Delta \chi$ [13-16,]. The energy capacity of the CSC and electrolytic UCS is determined by two main parameters. Discharge current, which is determined by polarization processes and operating voltage, which depends on the properties of the DEL.

3) According to equation (4), in order to increase the energy capacity of the CSC, the chemically active substance must be nanostructured in order to increase the number of dipole moments and their magnitude, and electrode materials must ensure the stable preservation of the original nanostructure during operation. One of the options for solving the problem is the use of a high-porous matrix in the creation of electrode materials. In this case, thin-film technology enables the creation of hybrid UCS, which integrates the properties of the CSC and UCS. Recently this approach is actively developed and shows good performance in terms of energy capacity (energy consumption 220-250-watt hour/kg)

4) The energy capacity of the CSC is determined by two main parameters (the electric capacity of the capacitor structure (C) and operating voltage of the capacitor $U$ ). The energy capacity of $\operatorname{CSC}\left(A_{i}\right)$ is determined by the formula $A_{i}=\frac{\mathrm{CU}^{2}}{2}$. The electric capacity (C) in CSC is determined by the properties of the double electric layer (DEL), which occurs at the boundary between the electrode material and the electrolyte for electrolytic CSC. Almost all the properties of DEL is determined mainly by three parameters: free electrons density $\left(\boldsymbol{\rho}_{e}\right)$, which is determined by the properties of the electrode material, dielectric permeability ( $\boldsymbol{E}_{s}$ ) in DEL, describing the properties of the electrolyte and electrode materials, and the work of the output $\left(\boldsymbol{W}_{B}\right)$, characterizing the interaction of both environments. Two of these parameters $\boldsymbol{P}_{e}$ and $\boldsymbol{W}_{B}$ can be measured experimentally and the third parameter $\varepsilon_{s}$ - is free [13-16]. Therefore, implementing a dielectric layer into the electrode material design can increase significantly the operating voltage, creating highvoltage CSC. The main characteristic of ceramics is the dielectric permeability $\varepsilon$, the value of which in nanostructured materials reaches $10^{8}$, and in monolithic materials at the level of $10^{4}[18,19]$. . The value of dielectric permeability increases due to the increase in the number of dipole moments and their magnitude.

5) The operating voltage of the ceramic layer is determined by the value of the breakdown voltage, which increases with a decrease in the non-connected charges in ceramics [17]. Therefore, there is no direct relationship between the breakdown voltage $U_{n}$ and $\varepsilon[17]$. Electroporcelain $\varepsilon=5-$ $8 \mathrm{U}_{\mathrm{br}} 30-32 \frac{\mathrm{MV}}{\mathrm{m}}$. Ultra porcelain $\varepsilon=8-8,8 \mathrm{U}_{\mathrm{br}} 30-$ $36 \frac{\mathrm{MV}}{\mathrm{m}}$. Active ceramics Barium Titan ate $\varepsilon \sim 10^{4}$, $\mathrm{U}_{\mathrm{br}} \sim 0,8 \frac{\mathrm{MV}}{\mathrm{m}}[20]$.

6) Because the mechanism of electric energy accumulation in the CSC and UCS from a physical point of view is the same, there is a possibility to combine design solutions and to create a unified electrode material for CSC and UCS on thinfilm technology. In this case, it is possible to increase the specific energy capacity of the energy storage device while increasing its specific power in a single design solution.

7) Therefore, the development of advanced design of CSC and SUCS should go in the following directions:

The development of unified nanostructured electrode material for CSC and UCS;

The development of nanostructured active mass for CSC;

The development of ceramics and polymer-ceramic materials with dielectric permeability

$\varepsilon>10^{6} \div 10^{8}$ и $\mathrm{U}_{\mathrm{br}}>0,01 \div 0,1 \frac{\mathrm{MV}}{\mathrm{m}} ;$

The development of new electrolytes, providing high $\mathcal{E}_{s}$, in DEL high one-way conductivity;

The creation of a new generation of separators with barrier layers that reduce leakage currents.

The work is carried out with the financial support of the state represented by the Ministry of Education and Science of Russia. Agreement of grant No. 14.577.21.0275 from 26.09.2017

\section{References}

[1] Blomgren, G. E. The Development and Future of Lithium Ion Batteries / G. E. Blomgren // Journal of the Electrochemical Society. 2017. - Vol. 164, Issue 1. - P. A5019-A5025. - DOI: 10.1149/2.0251701jes _ $\quad$ _ nttp://jes.ecsdl.org/content/164/1/A5019.full

[2] Myung, S. T. Nickel-Rich Layered Cathode Materials for Automotive Lithium-Ion Batteries: Achievements and Perspectives / S.-T. Myung, F. Maglia, K.-J. Park, C. S. Yoon, P. Lamp, S.-J. Kim, Y. K. Sun // ACS Energy Letters. - 2017. - Vol. 2. - P. 196-223. - DOI: 10.1021/acsenergylett.6b00594. http://pubs.acs.org/doi/pdfplus/10.1021/acsenergylett.6b00594

[3] Christiansen, C. Energy Storage Study: A Storage Market Review and Recommendations for Funding and Knowledge Sharing Priorities / C. Christiansen, B. Murray, G. Conway // AECOM Australia for Australian Renewable Energy Agency. - 13 July 2015. - P. 32. (http://arena.gov.au/files/2015/07/AECOM-Energy-StorageStudy.pdf).

[4] Kitsyuk E. P. "Research and development of processes for the formation of nanostructured electrodes of electrochemical energy storage devices", thesis for the degree of Candidate of Engineering Sciences (Ph.D.) 05.27.06 - technology and equipment for the production of semiconductors, materials and devices of electronic equipment MIET, Moscow, 2017

[5] Lebedev E. F. "Development of formation processes and study of properties of elements of heat release and energy storage for thermoelectric batteries", thesis for the degree of Candidate of Engineering Sciences (Ph.D.), specialty 05.27.06. MIET, Moscow, 2017 
[6] VU DYC KHOAN “STUDY AND DEVELOPMENT OF MULTILAYER THIN-FILM ELECTROLYTIC CELLS”, dissertation on competition of a scientific degree of Candidate of Engineering Sciences (Ph.D.), specialty 05.27.06 Moscow - 2017.

[7] Melvin-Hughes E.A. "Physical chemistry" Publishing house I.L. Moscow 1962

[8] Gerasimov Ya.I.; Dreving V.P.; Eremin E.N., etc. the general editorship of A.A. Gerasimov. "Course of physical chemistry", Chemisty Publishing house, Moscow 1969

[9] Prigozhin M. and Defay R. "Chemical thermodynamics". Publishing house "Binom", Moscow 2010.

[10] Fikhtengolts G.M. "Course of differential and integral calculation". Publishing house "Physico-mathematical literature" Moscow 1969

[11] Suzdalev I.P. "Nanotechnology: Physical chemistry of nanoclusters, nanostructures and nanomaterials" Ed. Moscow: Book house "Librokom", 2009.

[12] Sleptsov V.V. "Physico-chemical fundamentals of nanomaterials and nanotechnology". OOO "Sam poligrafist" Moscow 2015.

[13] R. R. Salem "Physical chemistry. The beginning of theoretical electrochemistry" Publisher: "Komkniga" 2010.

[14] B. B. Damaskin, O. A. Petriy, G. A. Tsirlina. "Electrochemistry" publishing house "Chemistry" 2010

[15] Lewis George, Randall.M "Chemical thermodynamics". Publishing house "ONTI Khimteoret" Moscow 1936

[16] Salem P. P. "The theory of the double layer". Publishing house Fizmatlit, Moscow, 2003

[17] Matveev A.N. "Electricity and magnetism". Publisher Lan Saint Petersburg-Moscow 2010

[18] Belyaev B.A., Drokin N. A. Impedance spectra of thin permalloy films with nanostructure, solid state Physics, 2012, vol.54, iss.2, pp. 340-346.

[19] B.I.Sedunov, D. A. Frank-Kamenetsky,"Dielectric permittivity of biological objects", Physical Sciences Success, April 1963, v. LXXIX, iss. Four p. 617-639.

[20] The reference book "Physical quantities" Publishing house Energoatomizdat, Moscow, 1991 